\title{
Self-reported smell and taste recovery in coronavirus disease 2019 patients: a one-year prospective study
}

\author{
Paolo Boscolo-Rizzo ${ }^{1}$ (D) - Francesco Guida ${ }^{1}$. Jerry Polesel ${ }^{2}$ - Alberto Vito Marcuzzo ${ }^{1}$ - Paolo Antonucci ${ }^{1}$. \\ Vincenzo Capriotti ${ }^{3}$ - Erica Sacchet ${ }^{1}$. Fiordaliso Cragnolini ${ }^{1} \cdot$ Andrea D'Alessandro $^{1}$ - Enrico Zanelli ${ }^{1}$. \\ Riccardo Marzolino ${ }^{1}$. Chiara Lazzarin ${ }^{1}$ - Margherita Tofanelli ${ }^{1} \cdot$ Nicoletta Gardenal $^{1}$ - Daniele Borsetto ${ }^{4}$. \\ Claire Hopkins $^{5}$. Luigi Angelo Vaira ${ }^{6} \cdot$ Giancarlo Tirelli $^{1}$
}

Received: 16 March 2021 / Accepted: 19 April 2021 / Published online: 7 May 2021

(c) The Author(s), under exclusive licence to Springer-Verlag GmbH Germany, part of Springer Nature 2021

\begin{abstract}
Purpose The aim of the present study was to estimate the 1 year prevalence and recovery rate of self-reported chemosensory dysfunction in a series of subjects with previous mild-to-moderate symptomatic COVID-19.

Methods Prospective study based on the SNOT-22, item "sense of smell or taste" and additional outcomes.

Results 268/315 patients (85.1\%) completing the survey at baseline also completed the follow-up interview. The 12 months prevalence of self-reported COVID-19 associated chemosensory dysfunction was $21.3 \%$ (95\% CI 16.5-26.7\%). Of the 187 patients who complained of COVID-19 associated chemosensory dysfunction at baseline, 130 (69.5\%; 95\% CI 62.4-76.0\%) reported complete resolution of smell or taste impairment, $41(21.9 \%)$ reported a decrease in the severity, and $16(8.6 \%)$ reported the symptom was unchanged or worse 1 year after onset. The risk of persistence was higher for patients reporting a baseline SNOT-22 score $\geq 4(\mathrm{OR}=3.32 ; 95 \% \mathrm{CI} 1.32-8.36)$ as well as for those requiring $\geq 22$ days for a negative swab $(\mathrm{OR}=2.18 ; 95 \%$ CI 1.12-4.27).

Conclusion A substantial proportion of patients with previous mild-to-moderate symptomatic COVID-19 characterized by new onset of chemosensory dysfunction still complained on altered sense of smell or taste 1 year after the onset.
\end{abstract}

Keywords COVID-19 $\cdot$ Anosmia $\cdot$ SARS-CoV-2 $\cdot$ Coronavirus $\cdot$ Smell $\cdot$ Taste $\cdot$ Outcome

Paolo Boscolo-Rizzo

paolo.boscolorizzo@units.it

1 Department of Medical, Surgical and Health Sciences, Section of Otolaryngology, University of Trieste, Trieste, Italy

2 Unit of Cancer Epidemiology, Centro di Riferimento Oncologico di Aviano (CRO) IRCCS, Aviano, Italy

3 Department of Neurosciences, Section of Otolaryngology, Papa Giovanni XXIII General Hospital, Bergamo, Italy

4 Department of ENT, Addenbrooke's Hospital, Cambridge University Hospitals NHS Foundation Trust, Cambridge, UK

5 Guy's and St Thomas' Hospitals, London, UK

6 Maxillofacial Surgery Operative Unit, Department of Medical, Surgical and Experimental Sciences, University of Sassari, Sassari, Italy

\section{Introduction}

One year after the outbreak of the coronavirus-19 disease (COVID-19) pandemic in Europe, alterations in smell and taste have unquestionably emerged as a highly prevalent symptom and the most reliable predictor of the severe acute respiratory syndrome-coronavirus-2 (SARS-CoV-2) infection [1-3]. In addition, a high persistence rate of chemosensory disorders has been reported 6 months after onset $[4,5]$. However, the results of these reports cannot be considered as definitive as in some forms of post-viral anosmia, recovery times of more than 1 year have been reported in the past [6].

Although psychophysical evaluation of the olfactory function has higher sensitivity, especially in detecting mild hyposmia [4, 7], the self-reported evaluation of chemosensitivity has a baseline parameter of comparison consisting in the subjective perception of smell or taste preceding the onset of COVID-19. In older adults, indeed, the prevalence 
of psychophysical olfactory impairment in the setting of no reported deficit is $15 \%$ [8].

The aim of the present study was to estimate the 1 year prevalence and recovery rate of self-reported chemosensory dysfunction in a series of subjects with previous mild-tomoderate symptomatic COVID-19.

\section{Materials and methods}

The study was approved by the Ethics Committee of the Friuli Venezia Giulia Region (CEUR-2020-Os-156), and informed consent was obtained verbally for telephone interviews.

\section{Subjects}

This is a prospective study on mild-to-moderate symptomatic adult patients consecutively assessed at Trieste University Hospital between March 1 and March 31, 2020, who tested positive for SARS-CoV-2 RNA by polymerase chain reaction (PCR) on nasopharyngeal and throat swabs performed according to World Health Organization recommendation [9]. All patients were initially home-isolated with mild-to-moderate symptoms. Patients were considered mild-to-moderate symptomatic if they had less severe clinical symptoms with no evidence of pneumonia, not requiring hospitalization, and therefore considered suitable for being treated at home. Patients with a history of previous trauma, surgery or radiotherapy in the oral and nasal cavities, allergic rhinitis or rhinosinusitis, previous olfactory or gustative dysfunction or psychiatric or neurological diseases, were excluded from the study.

$315(72.7 \%)$ of the 433 eligible patients completed the baseline telephone interview administrated within 3 weeks after the first positive swab performed between 1 st and 22nd March 2020. The median time from symptoms onset to SARS-CoV-2 testing was 7 days (interquartile range 4-11 days). All patients completing the baseline interview were phoned from 5 to 12th March 2021, so that all patients were recontacted 12 months after the onset of symptoms; in case of a non-response, patients were re-contacted twice.

\section{Questionnaires}

Demographic and clinical data were collected through ad hoc questions administered during the baseline interview and included gender, age, self-reported height and weight, smoking and alcohol habits, and the following co-morbidities: immunosuppression, diabetes, cardiovascular diseases, active cancer, chronic respiratory disease, kidney disease, liver disease. Obesity was defined as having a body mass index (BMI) of 30 or more. The sense of smell and taste was assessed by the sino-nasal outcome test 22 (SNOT-22) [10], item "sense of smell or taste", both at baseline and during the follow-up interview to evaluate their persistence and the recovery rate. The SNOT-22 grades symptom severity as none (0), very mild (1), mild or slight (2), moderate (3), severe (4), or as bad as it can be (5). Patients with SNOT$22>0$ were also asked whether the chemosensory alteration involved the sense of smell, taste, or both. Patients were finally asked about blocked nose based on the item scores No $=0$, Yes-mild-to-moderate $=1$, and Yes-severe $=2$. The dates of the first positive and negative swabs were obtained from hospital records.

\section{Statistical analysis}

Symptom prevalence was expressed as percentage of total patients, and 95\% confidence interval (CI) were calculated using Clopper-Pearson method; differences in prevalence were evaluated through Fisher's exact test. The risk of chemosensory impairment persistence, expressed as odds ratio (OR), was estimated through unconditional logistic regression model, adjusting for gender and age. Variables which were significant at the univariate analysis were further included in the multivariable model. Analyses were performed using R 3.6 and statistical significance was claimed for $p<0.05$ (two-tailed).

\section{Results}

Of 315 patients completing the survey at baseline, $47 \mathrm{did}$ not answer or refused the follow-up interview, thus leaving 268 responders $(85.1 \%)$. Baseline sociodemographic and clinical characteristics of 268 patients are reported in Table 1. The median age of the study cohort was 48 years (interquartile range, 38-56 years). There was a female preponderance with 166 out of 268 being females (61.9\%). Associated comorbidities were reported by 91 cases $(34.0 \%)$ with the most common being obesity reported by 32 patients (11.9\%) followed by cardiovascular diseases $(n=25,9.3 \%)$ and immunosuppression ( $n=19,7.1 \%)$.

The median time to achieving a negative swab was 22 days (interquartile range 15-31 days). Overall, 187 of 268 responders $(69.8 \%$, CI 95\% 63.9-75.2) reported an altered sense of smell or taste at baseline (SNOT-22>0) with 99 (36.9\%, CI 95\% 31.1-43.0) reporting the highest SNOT22 score, for severe problem. Particularly, 152 subjects (81.3\%) self-reported combined chemosensory dysfunction, $19(10.2 \%)$ patients self-reported isolated smell impairment and 16 patients (8.6\%) self-reported isolated taste disorder. No sociodemographic characteristics or clinical features were associated with either chemosensory dysfunctions or its severity at baseline (data not shown). 
Table 1 Baseline sociodemographic and clinical characteristics of 268 patients positive for SARS-CoV-2

\begin{tabular}{|c|c|c|c|}
\hline & $n$ & $\%$ & $(95 \% \mathrm{CI})$ \\
\hline \multicolumn{4}{|l|}{ Gender } \\
\hline Male & 102 & 38.1 & $(32.2-44.2)$ \\
\hline Female & 166 & 61.9 & $(55.8-27.8)$ \\
\hline \multicolumn{4}{|l|}{ Age (years) } \\
\hline$<40$ & 79 & 29.5 & $(24.1-35.3)$ \\
\hline $40-54$ & 103 & 38.4 & $(32.6-44.5)$ \\
\hline$\geq 55$ & 86 & 32.1 & $(26.5-38.0)$ \\
\hline \multicolumn{4}{|l|}{ Smoking habits } \\
\hline Never & 157 & 58.6 & $(52.4-64.5)$ \\
\hline Former & 68 & 25.4 & $(20.3-31.0)$ \\
\hline Current & 43 & 16.0 & $(11.9-21.0)$ \\
\hline \multicolumn{4}{|l|}{ Drinking habits } \\
\hline Never & 176 & 65.7 & $(59.7-71.3)$ \\
\hline Former & 27 & 10.1 & $(6.7-14.3)$ \\
\hline Current & 65 & 24.3 & $(19.2-29.8)$ \\
\hline \multicolumn{4}{|l|}{$\operatorname{BMI}\left(\mathrm{kg} \mathrm{m}^{-2}\right)$} \\
\hline$<25$ & 146 & 54.5 & $(48.3-60.5)$ \\
\hline $25-29.9$ & 84 & 31.3 & $(25.8-37.3)$ \\
\hline$\geq 30$ & 38 & 14.2 & $(10.2-18.9)$ \\
\hline \multicolumn{4}{|l|}{ Comorbidity } \\
\hline None & 177 & 66.0 & $(60.0-76.0)$ \\
\hline Any & 91 & 34.0 & $(28.3-40.0)$ \\
\hline \multicolumn{4}{|l|}{ Blocked nose } \\
\hline 0: No & 218 & 81.3 & $(76.2-85.8)$ \\
\hline 1: Yes—mild-to-moderate & 35 & 13.1 & $(9.3-17.7)$ \\
\hline 2: Yes-severe & 15 & 5.6 & $(3.2-9.1)$ \\
\hline \multicolumn{4}{|l|}{ Chemosensory dysfunction } \\
\hline No & 81 & 30.2 & $(24.8-36.1)$ \\
\hline Yes & 187 & 69.8 & $(63.9-75.2)$ \\
\hline \multicolumn{4}{|c|}{ Type of chemosensory dysfunction } \\
\hline None & 81 & 30.2 & $(24.8-36.1)$ \\
\hline Smell & 19 & 7.1 & $(4.3-10.9)$ \\
\hline Taste & 16 & 6.0 & $(3.5-9.5)$ \\
\hline Smell and taste & 152 & 56.7 & $(50.6-62.7)$ \\
\hline \multicolumn{4}{|l|}{ SNOT-22 at diagnosis ${ }^{\mathrm{a}}$} \\
\hline 0: No & 81 & 30.2 & $(24.8-36.1)$ \\
\hline 1: Very mild & 3 & 1.1 & $(0.2-3.2)$ \\
\hline 2: Mild or slight & 18 & 6.7 & $(4.0-10.4)$ \\
\hline 3: Moderate & 27 & 10.1 & $(6.7-14.3)$ \\
\hline 4: Severe & 40 & 14.9 & $(10.9-19.8)$ \\
\hline 5: As bad as it can be & 99 & 36.9 & $(31.1-43.0)$ \\
\hline \multicolumn{4}{|l|}{ Time for negative swab (days) } \\
\hline$<22$ & 128 & 47.8 & $(41.6-53.9)$ \\
\hline$\geq 22$ & 140 & 52.2 & $(46.1-58.4)$ \\
\hline
\end{tabular}

SARS-CoV-2 severe acute respiratory syndrome coronavirus

aAccording to sino-nasal outcome test 22 (SNOT-22), item "sense of smell or taste"
After 12 months, 57 patients (21.3\%; 95\% CI 16.5-26.7\%) still reported chemosensory dysfunction, with 34 subjects still reporting both smell and taste dysfunction, 15 reporting smell impairment and 5 taste disorder. Among patients with persistent chemosensory dysfunction, only 4 (7.0\%) complained blocked nose. Among the 187 patients who have complained a COVID-19 associated chemosensory dysfunction at baseline, 130 (69.5\%; 95\% CI 62.4-76.0\%) reported complete resolution of smell or taste impairment, 41 (21.9\%; 95\% CI 16.2-28.5\%) reported a decrease in the severity, and 16 (8.6\%; 95\% CI 5.0-13.5\%) reported the symptom was unchanged or worse (Table 2). Baseline sociodemographic and lifestyle factors were not associated with the persistence of chemosensory dysfunction (Table 3). After adjustment for covariates, the severity of chemosensory dysfunction at baseline (OR $=3.32$; $95 \%$ CI $1.32-8.36$ for SNOT-22 score $\geq 4)$ and a longer time to achieving a negative swab $(\mathrm{OR}=2.18 ; 95 \%$ CI $1.12-4.27)$ were associated with a higher risk of persistence of symptoms at 12 months.

\section{Discussion}

Among the 187 patients who have complained a COVID-19 associated chemosensory dysfunction, $69.5 \%$ experienced a complete resolution of these symptoms at 12 months, while $30.5 \%$ still reported impairment in their chemosensory function. Thus, the 12 months overall prevalence of persistent altered sense of smell or taste in this series of subjects with previous mild-to-moderate symptomatic COVID-19 was $21.3 \%$.

To the best of our knowledge, this is the first study to estimate the 12 months prevalence and recovery rate of selfreported chemosensory dysfunction in a series of subjects with previous mild-to-moderate symptomatic SARS-CoV-2 infection. Such a long follow-up is essential to estimate the real prevalence of COVID-19 related chemosensory disorders as functional recoveries after 1 year from infection with other viruses have been reported in the past even [6].

The data from our study highlight a significant rate of lasting olfactory dysfunction as a legacy of the pandemic. Considering the spread out of SARS-CoV-2 infections in Europe with more than $36,000,000$ cases to date [11], the burden of chemosensory disorders on the health systems will be even more pressing. Indeed, given that our estimates are based on self-reported symptoms, these patients will likely seek medical care for their chemosensory disorders.

This prevalence is likely underestimated compared to the rate that would have been obtained using the most accurate psychophysical evaluation $[4,12]$. We have previously shown that in a cohort of patients self-reporting normal 
Table 2 Change in alteration of sense of smell or taste in 268 patients positive for SARS-CoV-2

\begin{tabular}{|c|c|c|c|c|c|c|c|}
\hline \multirow[t]{2}{*}{ SNOT-22 at baseline ${ }^{a}$} & \multicolumn{6}{|c|}{ SNOT-22 after 12 months ${ }^{\mathrm{a}}$} & \multirow[t]{2}{*}{ Total } \\
\hline & 0: No & 1: Very mild & 2: Mild or slight & 3: Moderate & 4: Severe & 5: As bad as it can be & \\
\hline 0: No & 81 & 0 & 0 & 0 & 0 & 0 & 81 \\
\hline 1: Very mild & 3 & 0 & 0 & 0 & 0 & 0 & 3 \\
\hline 2: Mild or slight & 18 & 0 & 0 & 0 & 0 & 0 & 18 \\
\hline 3: Moderate & 20 & 2 & 2 & 1 & 1 & 1 & 27 \\
\hline 4: Severe & 30 & 3 & 4 & 1 & 2 & 0 & 40 \\
\hline 5: As bad as it can be & 59 & 7 & 12 & 10 & 7 & 4 & 99 \\
\hline Total & 211 & 12 & 18 & 12 & 10 & 5 & \\
\hline$\%$ & 78.7 & 4.5 & 6.7 & 4.5 & 3.7 & 1.9 & \\
\hline$(95 \% \mathrm{CI})$ & $(73.3-83.5)$ & $(2.3-7.7)$ & $(4.0-10.4)$ & $(2.3-7.7)$ & $(1.8-6.8)$ & $(0.6-4.3)$ & \\
\hline
\end{tabular}

SARS-CoV-2 severe acute respiratory syndrome coronavirus

according to sino-nasal outcome test 22 (SNOT-22), item "sense of smell or taste"

sense of smell 6 months after previous SARS-CoV-2 infection, psychophysical testing revealed olfactory deficits in $59 \%$, including anosmia in 5\% [4]. However, this subjective evaluation may be more accurate in estimating the percentage of patients in whom the smell or taste disturbance has repercussions on their quality of life. Furthermore, the evaluation with psychophysical tests alone could overestimate the prevalence of residual COVID-19-related chemosensory disorders including all those subjects who were unaware of having a previous olfactory or gustatory dysfunction [8]. Nevertheless, self-rating of the olfactory function is imprecise and a psychophysical evaluation is essential to corroborate the patient's complaints and measure the degree of smell and taste impairment [13]. Also, as olfaction plays a critical role in determining the appreciation of flavor in foods and beverages and many patients fail to distinguish between taste and flavor, a self-assessment of chemosensory function has the potential of smell and taste confusion [14]. It may also be important to accurately measure the residual olfactory function in patients who have yet not fully recovered as this assessment may serve as a good prognostic predictor [15].

These observations should prompt the experts in chemosensory disorders to make further efforts to test possible treatments for post-COVID-19 smell and taste disorders. Current therapies are essentially based on the experience gained in the study of other forms of post-viral anosmia. Recently, the clinical olfactory working group members made an overwhelming recommendation for olfactory training for post-viral anosmia including COVID-19 [16]. However, it is imperative to design multicentre clinical trials to test olfactory training efficacy as well as new therapeutic strategies for improving chemosensory outcomes in patients with post-COVID-19 chemosensory disorders. The high number of potentially recruitable patients offers a unique opportunity to test the efficacy of new therapeutic approaches for post-viral chemosensory disorders.
We found that the severity of chemosensory dysfunction at baseline was associated with a higher risk of persistence of symptoms at 12 months. Thus, patients with more severe smell or taste impairment should be preferentially included in randomized clinical trials evaluating the efficacy of different treatment approaches, and research is required to determine if treatment at an early stage can reduce the rate of persistent dysfunction.

We also found that a longer duration of viral persistence on PCR testing was associated with an increased risk of persistent chemosensory dysfunction. It has been shown that SARS-CoV-2 gains entry to the supporting cells of the respiratory epithelium [17]. It could be hypothesized that persistence of viral infection in the nose prevents recovery of the supporting cells, which leads indirectly to injury of the olfactory sensory neurones and persistent loss. In contrast, rapid viral clearance may allow repair of the integrity of the olfactory epithelium before loss of the olfactory sensory neurons occurs.

Interestingly, among patients with persistent chemosensory dysfunction, only $7.0 \%$ complained blocked nose. This is consistent with previous investigations showing that in contrast to other acute viral smell impairment, COVID19-associated smell loss is only rarely accompanied by a severely blocked nose $[18,19]$. This observation highlights the potential predictive value of an altered sense of smell within the COVID-19 pandemic context.

The data from the present study should be taken cautiously owing to several study limitations. Symptoms were self-reported, based on cross-sectional surveys, and may therefore contain suboptimal sensitivity. Subjective evaluation of the olfactory function was observed, indeed, to be inadequate to fully evaluate olfactory recovery with several authors having underlined that subjectivity of self-reporting may lead to underestimation of the prevalence of olfactory dysfunction. It is clear that patients' rating of whether smell 
Table 3 Odds ratio $(\mathrm{OR})$ and $95 \%$ confidence interval $(\mathrm{CI})$ for persistence or worsening at 12 months of alteration of sense of smell or taste according to baseline characteristics

\begin{tabular}{|c|c|c|c|c|c|c|}
\hline \multirow[t]{2}{*}{ Characteristics } & \multicolumn{2}{|c|}{$\begin{array}{l}\text { Persistence or } \\
\text { worsening }\end{array}$} & \multicolumn{2}{|c|}{ Recovered } & \multirow[t]{2}{*}{ OR $(95 \% \mathrm{CI})^{\mathrm{a}}$} & \multirow[t]{2}{*}{ OR $(95 \% \mathrm{CI})^{\mathrm{b}}$} \\
\hline & $n$ & $(\%)$ & $n$ & $(\%)$ & & \\
\hline \multicolumn{7}{|l|}{ Gender } \\
\hline Male & 17 & $(29.8)$ & 53 & $(40.8)$ & Reference & Reference \\
\hline Female & 40 & $(70.2)$ & 77 & $(59.2)$ & $1.67(0.85-3.27)$ & $1.83(0.90-3.72)$ \\
\hline \multicolumn{7}{|l|}{ Age (years) } \\
\hline$<40$ & 20 & $(35.1)$ & 35 & $(26.9)$ & Reference & Reference \\
\hline $40-54$ & 21 & $(36.8)$ & 55 & $(42.3)$ & $0.64(0.30-1.35)$ & $0.49(0.22-1.11)$ \\
\hline$\geq 55$ & 19 & $(28.1)$ & 40 & $(30.8)$ & $0.69(0.31-1.54)$ & $0.59(0.25-1.38)$ \\
\hline \multicolumn{7}{|l|}{ Smoking habits } \\
\hline Never & 30 & $(52.6)$ & 84 & $(64.6)$ & Reference & Reference \\
\hline Ever & 27 & $(47.4)$ & 46 & $(35.4)$ & $1.77(0.93-3.38)$ & $1.77(0.89-3.51)$ \\
\hline \multicolumn{7}{|l|}{ Drinking habits } \\
\hline Never & 42 & $(73.7)$ & 79 & $(60.8)$ & Reference & Reference \\
\hline Ever & 15 & $(26.3)$ & 41 & $(39.2)$ & $0.60(0.29-1.21)$ & $0.67(0.32-1.42)$ \\
\hline \multicolumn{7}{|l|}{ BMI $\left(\mathrm{kg} \mathrm{m}^{-2}\right)$} \\
\hline$<30$ & 35 & $(61.4)$ & 59 & $(45.4)$ & Reference & Reference \\
\hline$\geq 30$ & 22 & $(38.6)$ & 71 & $(54.6)$ & $0.72(0.30-1.75)$ & $0.55(0.22-1.40)$ \\
\hline \multicolumn{7}{|l|}{ Blocked nose } \\
\hline No & 40 & $(70.2)$ & 107 & $(82.3)$ & Reference & Reference \\
\hline Yes & 17 & $(29.8)$ & 23 & $(17.7)$ & $1.97(0.95-4.11)$ & $2.14(0.98-4.67)$ \\
\hline \multicolumn{7}{|l|}{ Comorbidity } \\
\hline None & 39 & $(68.4)$ & 81 & $(62.3)$ & Reference & Reference \\
\hline Any & 18 & $(31.6)$ & 49 & $(37.7)$ & $0.80(0.41-1.59)$ & $0.84(0.40-1.74)$ \\
\hline \multicolumn{7}{|c|}{ Type of chemosensory impairment } \\
\hline Smell or taste & 5 & $(8.8)$ & 30 & $(23.1)$ & Reference & Reference \\
\hline Smell and taste & 52 & $(91.2)$ & 100 & $(76.9)$ & $3.37(1.22-9.33)$ & $2.58(0.91-7.37)$ \\
\hline \multicolumn{7}{|l|}{ SNOT-22 ${ }^{b}$} \\
\hline $1-3$ & 7 & $(12.3)$ & 41 & $(31.5)$ & Reference & Reference \\
\hline $4-5$ & 50 & $(87.7)$ & 89 & $(68.5)$ & $3.84(1.55-9.49)$ & $3.32(1.32-8.36)$ \\
\hline \multicolumn{7}{|c|}{ Time for negative swab (days) } \\
\hline$<22$ & 21 & $(36.8)$ & 70 & $(53.9)$ & Reference & Reference \\
\hline$\geq 22$ & 36 & $(63.2)$ & 60 & $(46.2)$ & $2.09(1.09-3.99)$ & $2.18(1.12-4.27)$ \\
\hline
\end{tabular}

$B M I$ body mass index

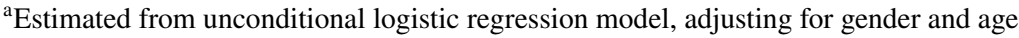

${ }^{b}$ Further adjusted for smell/taste alteration, severity of alteration, and time for negative swab

bAccording to sino-nasal outcome test 22 (SNOT-22), item "sense of smell or taste" and or taste are impacted differentially, is very difficult to interpret, due to impact of loss perception of flavor through retronasal olfaction, and little can be inferred from this aspect of the study. Moreover, the study cohort was relatively small and patients with more severe COVID-19 were excluded. Finally, as recovery in other post-infection olfactory disorders was observed to take from 2 to 3 years [20], these data should be considered as intermediate findings.

In conclusion, a substantial proportion of patients with previous mild-to-moderate symptomatic COVID-19 characterized by new onset of chemosensory dysfunction, still complained on altered sense of smell or taste 1 year after the onset. As recovery from post-viral loss may continue beyond this period $[6,21]$, further assessments will be necessary to conclude whether smell and taste dysfunction is permanent. Moreover, there is urgent need for more efforts and research on treatment strategies for post-COVID-19 chemosensory dysfunction.

Author contributions PB-R and FG had full access to all of the data in the study and take responsibility for the integrity of the data and the accuracy of the data analysis. Concept and design: B-R, FG, CH, LAV and GT. Acquisition or interpretation of data: PB-R, FG, AVM, PA, VC, ES, FC, AD'A, EZ, RM, CL, MT, NG, DB. Drafting of the 
manuscript: PB-R, FG, JP, CH, LAV, GT. Critical revision of the manuscript for important intellectual content: PB-R, FG, JP, DB, CH, LAV, GT. Statistical analysis: JP. Supervision: PB-R, CH, LAV, and GT.

Funding None.

Availability of data and material The authors confirm that the data supporting the findings of this study are available within the article.

\section{Declarations}

Conflict of interest The authors declare that they have no conflict of interest.

Ethical approval The study was approved by the Ethics Committee of the Friuli Venezia Giulia Region (CEUR-2020-Os-156).

Informed consent Additional informed consent was obtained from all individual participants for whom identifying information is included in this article.

\section{References}

1. Lechien JR, Chiesa-Estomba CM, De Siati DR, Horoi M, Le Bon SD, Rodriguez A et al (2020) Olfactory and gustatory dysfunctions as a clinical presentation of mild-to-moderate forms of the coronavirus disease (COVID-19): a multicenter European study. Eur Arch Oto-Rhino-Laryngol. https://doi.org/10.1007/ s00405-020-05965-1

2. Spinato G, Fabbris C, Polesel J, Cazzador D, Borsetto D, Hopkins $\mathrm{C}$ et al (2020) Alterations in smell or taste in mildly symptomatic outpatients with SARS-CoV-2 infection. JAMA. https://doi.org/ 10.1001/jama.2020.6771

3. Gerkin RC, Ohla K, Veldhuizen MG, Joseph PV, Kelly CE, Bakke AJ et al (2020) Recent smell loss is the best predictor of COVID19 among individuals with recent respiratory symptoms. Chem Senses. https://doi.org/10.1093/chemse/bjaa081

4. Boscolo-Rizzo P, Menegaldo A, Fabbris C, Spinato G, Borsetto D, Vaira LA et al (2021) Six-month psychophysical evaluation of olfactory dysfunction in patients with COVID-19. Chem Senses. https://doi.org/10.1093/chemse/bjab006

5. Hopkins C, Surda P, Vaira LA, Lechien JR, Safarian M, Saussez $S$ et al (2020) Six month follow-up of self-reported loss of smell during the COVID-19 pandemic. Rhinology. https://doi.org/10. 4193/Rhin20.544

6. Lee DY, Lee WH, Wee JH, Kim J-W (2014) Prognosis of postviral olfactory loss: follow-up study for longer than one year. Am J Rhinol Allergy 28:419-422. https://doi.org/10.2500/ajra.2014. 28.4102

7. Vaira LA, Deiana G, Fois AG, Pirina P, Madeddu G, De Vito A et al (2020) Objective evaluation of anosmia and ageusia in COVID-19 patients: single-center experience on 72 cases. Head Neck. https://doi.org/10.1002/hed.26204

8. Murphy C, Schubert CR, Cruickshanks KJ, Klein BEK, Klein R, Nondahl DM (2002) Prevalence of olfactory impairment in older adults. JAMA 288:2307-2312. https://doi.org/10.1001/jama.288. 18.2307
9. Technical guidance n.d. https://www.who.int/emergencies/disea ses/novel-coronavirus-2019/technical-guidance. Accessed Apr 27 2020

10. Hopkins C, Gillett S, Slack R, Lund VJ, Browne JP (2009) Psychometric validity of the 22 -item sinonasal outcome test. Clin Otolaryngol 34:447-454. https://doi.org/10.1111/j.1749-4486. 2009.01995.x

11. Coronavirus Update (Live): $120,126,411$ cases and 2,661,042 deaths from COVID-19 virus pandemic-worldometer n.d. https://www.worldometers.info/coronavirus/. Accessed Mar 14 2021

12. Vaira LA, Hopkins C, Petrocelli M, Lechien JR, Chiesa-Estomba CM, Salzano G et al (2020) Smell and taste recovery in coronavirus disease 2019 patients: a 60-day objective and prospective study. J Laryngol Otol 134:703-709. https://doi.org/10.1017/ S0022215120001826

13. Landis BN, Hummel T, Hugentobler M, Giger R, Lacroix JS (2003) Ratings of overall olfactory function. Chem Senses 28:691-694. https://doi.org/10.1093/chemse/bjg061

14. Deems DA, Doty RL, Settle RG, Moore-Gillon V, Shaman P, Mester AF et al (1991) Smell and taste disorders, a study of 750 patients from the University of Pennsylvania smell and taste center. Arch Otolaryngol Head Neck Surg 117:519-528. https:// doi.org/10.1001/archotol.1991.01870170065015

15. Hummel T, Lötsch J (2010) Prognostic factors of olfactory dysfunction. Arch Otolaryngol Head Neck Surg 136:347-351. https:// doi.org/10.1001/archoto.2010.27

16. Addison AB, Wong B, Ahmed T, Macchi A, Konstantinidis I, Huart $\mathrm{C}$ et al (2021) Clinical olfactory working group consensus statement on the treatment of postinfectious olfactory dysfunction. J Allergy Clin Immunol. https://doi.org/10.1016/j.jaci.2020.12. 641

17. Brann DH, Tsukahara T, Weinreb C, Lipovsek M, Van den Berge $\mathrm{K}$, Gong B et al (2020) Non-neuronal expression of SARS-CoV-2 entry genes in the olfactory system suggests mechanisms underlying COVID-19-associated anosmia. Sci Adv. https://doi.org/10. 1126/sciadv.abc5801

18. Haehner A, Draf J, Dräger S, de With K, Hummel T (2020) Predictive value of sudden olfactory loss in the diagnosis of COVID19. J Oto-Rhino-Laryngol 82:175-180. https://doi.org/10.1159/ 000509143

19. Boscolo-Rizzo P, Borsetto D, Fabbris C, Spinato G, Frezza D, Menegaldo A et al (2020) Evolution of altered sense of smell or taste in patients with mildly symptomatic COVID-19. JAMA Otolaryngol. https://doi.org/10.1001/jamaoto.2020.1379

20. London B, Nabet B, Fisher AR, White B, Sammel MD, Doty RL (2008) Predictors of prognosis in patients with olfactory disturbance. Ann Neurol 63:159-166. https://doi.org/10.1002/ana. 21293

21. Reden J, Mueller A, Mueller C, Konstantinidis I, Frasnelli J, Landis BN et al (2006) Recovery of olfactory function following closed head injury or infections of the upper respiratory tract. Arch Otolaryngol 132:265-269. https://doi.org/10.1001/archotol. 132.3.265

Publisher's Note Springer Nature remains neutral with regard to jurisdictional claims in published maps and institutional affiliations. 